\title{
EVALUACIÓN DE LA ACTIVIDAD ALELOPÁTICA DEL EXTRACTO EN ACETATO DE ETILO DE Miconia caudata (BONPL.) DC
}

\author{
ALLELOPATHIC ACTIVITY EVALUATION OF ETHYL ACETATE EXTRACT FROM Miconia caudata (BONPL.) \\ DC
}

\author{
Luz S. RAMÍREZ ARISTIZABAL**, Angélica M. MONTES ORTíz', José G. \\ MARTÍN BEDOYA', Francisco J. JIMÉNEZ-GONZÁLEZ ${ }^{1}$ \\ ${ }^{1}$ Grupo Polifenoles, Escuela de Tecnología Química, Facultad de Tecnología, Universidad \\ Tecnológica de Pereira. Pereira, Colombia.
}

*Autor Corresponsal. E-mail: luramire@utp.edu.co
Historia del Artículo

Recibido: Julio 26, 2016

Evaluado: Enero 05, 2017

Aceptado: Enero 21, 2017

Disponible: Febrero 09, 2017

Resumen |

El objetivo de este trabajo fue evaluar el potencial alelopático del extracto en acetato de etilo (AcOEt) y sus fracciones obtenidas de las hojas de Miconia caudata (Melastomataceae), a través del bioensayo de germinación y crecimiento en semillas de Lactuca sativa L. Inicialmente, el extracto en ACOEt se separó en columna cromatográfica sobre DIAION HP-20, utilizando un sistema de elución agua-isopropanol, obteniendo 20 fracciones las cuales fueron monitoreadas mediante la medición de la absorbancia a $280 \mathrm{~nm}$ y por cromatografía en capa delgada en fase normal y reversa. Adicionalmente, se realizaron pruebas químicas para la identificación de diferentes núcleos fitoquímicos. Los resultados obtenidos mostraron que la fracción F1M presentó un efecto estimulador de crecimiento en el hipocótilo con un máximo valor de $54.39 \%$, dicho efecto se puede atribuir a la presencia de taninos condensados. Las fracciones F1L y F1N tuvieron un comportamiento de actividad alelopática contrario, inhibiendo el crecimiento del hipocótilo de L. sativa. La fracción F1R presentó un mayor efecto inhibidor sobre las plántulas, con valores máximos de $86.67 \%$ y $93.78 \%$. A la fracción F1R se le realizaron pruebas químicas que indicaron la presencia de lactonas sesquiterpénicas, las cuales pueden estar involucradas en la actividad alelopática presentada.

Palabras Clave: Alelopatía, lactonas sesquiterpénicas, Lactuca sativa, Miconia caudata, taninos.

\section{Abstract}

The objective of this work was to evaluate the allelopathic potential of the extract in ethyl acetate (EtOAc) and its fractions obtained from the leaves of Miconia caudata (Melastomataceae), through the bioassay of germination and growth in seeds of Lactuca sativa L. Initially the extract in EtOAc was separated on a chromatographic column on DIAION HP-20, using a waterisopropanol elution system, obtaining 20 fractions which were monitored by measuring the absorbance at $280 \mathrm{~nm}$ and by thin layer chromatography normal and reverse phase. In addition, chemical test were performed for the identification of differents phytochemical core. The results obtained showed that the F1M fraction had a stimulating effect on the hypocotyl with a maximum value of $54.39 \%$, this effect can be attributed to the presence of condensed tannins. The fractions F1L and F1N had a behavior of opposite allelopathic activity, inhibiting the growth of the hypocotyl of L. sativa. The F1R fraction had a greater inhibitory effect on the seedlings, with maximum values of $86.67 \%$ and $93.78 \%$. The F1R fraction was subjected to chemical tests that indicated the presence of sesquiterpene lactones, which may be involved in the presented allelopathic.

Keywords: Allelopathy, Lactuca sativa, Miconia caudata, sesquiterpene lactones, tannins.

\section{INTRODUCCIÓN |}

Tanto en los ecosistemas naturales como en los agroecosistemas, las plantas liberan al medio una cantidad apreciable de compuestos biológicamente activos y algunos de ellos tienen efectos positivos o negativos sobre el crecimiento de las plantas que se encuentran en su entorno (Cutler \& Cutler, 2004). Estas sustancias se denominan aleloquímicos y su acción se conoce como alelopatía o efecto alelopático (Rice, 1984).

En las últimas décadas, la salud humana se ha visto afectada por la utilización indiscriminada de pesticidas para el control de malezas y plagas en cultivos. Es por esto, que ha adquirido especial importancia el manejo integral de los cultivos, y la búsqueda de compuestos naturales que 
disminuyan la utilización de plaguicidas sintéticos mediante la aplicación de sustancias de origen vegetal amigables al medio ambiente (Macías et al, 2004; Macías et al, 2007). Como parte de la búsqueda de este tipo de sustancias de origen vegetal que puedan servir en el control de malezas. Se analizó la actividad alelopática del extracto en acetato de etilo (AcOEt) de la planta Miconia caudata (melastomataceae) y sus fracciones, evaluando su efecto sobre Lactuca sativa L.

\section{MATERIALES Y MÉTODOS}

\section{Obtención del extracto y fracciones}

Las hojas de la planta Miconia caudata (melastomataceae) fueron recolectadas en el Jardín Botánico de la Universidad Tecnológica de Pereira (PereiraRisaralda), e identificada en el Herbario Nacional de Colombia (COL 520329). Las hojas se secaron a temperatura ambiente por 15 días, se molieron en molino de aspas (MF 10 basic IKA Werke) y se almacenaron en frascos ámbar en un lugar fresco.

Se tomaron $1500 \mathrm{~g}$ de hojas secas y molidas de Miconia caudata las cuales fueron desengrasadas con diclorometano (6 L); posteriormente el homogenizado se filtró y se concentró en rotaevaporador al vacío. El residuo obtenido de la extracción anterior se redisolvió en la mezcla isopropanol-agua (65:35) (6 L), se filtró y se concentró en rotaevaporador al vacío, hasta disminuir el volumen a la mitad para luego realizar una separación líquido-líquido con acetato de etilo (AcOEt) (Escobar et al., 2008). La fase en AcOEt se concentró al vacío hasta sequedad (8.30 g) y se le realizó separación en columna cromatográfica empacada con DIAION HP-20, eluyendo con gradiente agua-isopropanol (95:5;10:90). De este fraccionamiento se recogieron 150 fracciones, las cuales se agruparon en 20 subfracciones (F1AF1T), de acuerdo a sus perfiles cromatográficos por TLC (Waksmundzka-Hajnos et al., 2008) y medida de la absorbancia a $280 \mathrm{~nm}$. Posteriormente, se seleccionaron las fracciones de mayor interés de acuerdo a sus perfiles cromatográficos siendo llevadas hasta sequedad mediante rotaevaporación al vacío. A estas fracciones se les realizaron los estudios fitoquímicos y de actividad alelopática.

\section{Evaluación de la actividad alelopática}

Para la determinación de la actividad alelopática se utilizó la metodología propuesta por Galván et al, 2007 con algunas modificaciones. Se seleccionó la especie Lactuca sativa L. (lechuga), variedad Black Seeded Simpson como especie receptora estándar para el ensayo por ser más sensible a los diferentes aleloquímicos y por el corto período para su germinación y crecimiento. Las semillas se obtuvieron de la casa comercial Fercon Ltda. A las plántulas de lechuga se les realizó la medición (en $\mathrm{mm}$ ) del epicótilo e hipocótilo cada 24 horas por 7 días. Como control de inhibición del crecimiento se utilizó glifosato comercial (480
g/L, Roundup Spectra). Se preparó una solución de glifosato a una concentración de $3.8 \times 10^{-2} \mathrm{M}$. Como blanco se utilizó una mezcla de agua-DMSO al $0.33 \%$. Las soluciones se prepararon tomando $25 \mathrm{mg}$ del extracto disueltos en $0.1 \mathrm{~mL}$ de DMSO, posteriormente se completó a $25 \mathrm{~mL}$ con agua destilada previamente calentada hasta ebullición, de esta forma se utilizó un mínimo de muestra a una concentración de $1 \mathrm{mg} / \mathrm{mL}$. Las fracciones se prepararon tomando $8 \mathrm{mg}$ de fracción disueltos en $0.1 \mathrm{~mL}$ de DMSO, obteniéndose una concentración final de $1 \mathrm{mg} / \mathrm{mL}$. Las semillas previa desinfección se transfirieron a cajas de Petri sobre papel filtro y agua destilada previamente llevada hasta ebullición para su germinación durante 48 horas. Luego, se seleccionaron las semillas germinadas con radícula de $1 \mathrm{~mm}$ de longitud, para ser evaluadas frente al blanco, control y muestras. Las semillas germinadas seleccionadas se colocaron sobre papel de filtro, se les adicionó el volumen correspondiente para cada uno de los grupos de ensayo. Grupo 1 (blanco) con $30 \mathrm{~mL}$ de agua pretratada, grupo 2 (extractos y fracciones) con $25 \mathrm{~mL}$ de solución del extracto o fracción y grupo y grupo 3 (control) con $45 \mathrm{~mL}$ de solución de glifosato $\left(3.8 \times 10^{-2} \mathrm{M}\right)$. Para fracciones se utilizaron cajas con papel de filtro y 10 semillas. Los ensayos se realizaron por triplicado, durante 7 días. El porcentaje de actividad alelopática (\%A.A) se calculó de acuerdo a la expresión [(VPC.muestra-VPC.control)/(VPC.blanco-VPC.control)]x100; donde VPC es el valor promedio de crecimiento (en $\mathrm{mm}$ ). El \%A.A se presenta como efecto promotor de crecimiento dado en valores positivos y efecto inhibidor de crecimiento dado en valores negativos. Con las mediciones de la longitud de epicótilo e hipocótilo se calcularon el promedio y desviación estándar para cada tratamiento cada 24 h durante los 7 días del ensayo. Todos los ensayos se realizaron por triplicado.

\section{Fraccionamiento en columna cromatográfica}

Para la separación cromatográfica en columna sobre silica gel de la fracción F1R se utilizó gradiente en n-hexanoacetona, variando la proporción de los solventes inicialmente en 5\%, empezando en 95:5 hasta llegar a 80:20; y luego se cambió a 10\% hasta llegar a 100\% en acetona; luego se cambió el sistema a acetona-metanol variando la proporción de los solventes inicialmente en 5\%, empezando en 95:5 hasta llegar a 80:20; y luego se cambió a 10\% hasta llegar a 100\% metanol. De este fraccionamiento se recogieron 288 fracciones a las que se les realizó cromatografía en capa fina. Teniendo en cuenta este criterio, fueron agrupadas en 6 nuevas fracciones, rotuladas desde F1R1 hasta F1R6.

Con el fin de confirmar la presencia de compuestos de tipo tanino (Harborne, 1973; Schofield et al, 2001) se realizaron las pruebas en placa cromatografica en fase reversa con un sistema de elución n-butanol-ácido acéticoagua (BAW; 5:4:1) utilizando los agentes reveladores vainillina/ $\mathrm{HCl}, \mathrm{KIO}_{3}$ y $\mathrm{NaNO}_{2}$. Las pruebas de caracterización fitoquímica se realizaron tomando $0.1 \mathrm{mg}$ de cada muestra 
disuelta en metanol. Se transfirió la solución a una celda de cuarzo que fue llevada al espectrofotómetro UV/vis para realizar un barrido espectral. Para realizar las reacciones de desplazamiento se utilizaron los reactivos metilato sódico ( $\mathrm{MeONa}$ ), cloruro de aluminio y la mezcla de éste con $\mathrm{HCl}$. Los perfiles por cromatografía de capa delgada para las fracciones F1L, F1M, F1N y el extracto en AcOEt se realizaron utilizando el sistema de elución agua-isopropanol (7:3) en placas de fase reversa. Para el caso de la fracción F1R, se utilizaron placas de fase normal en el sistema de elución $n$ hexano-acetona (7:3). Para evidenciar la presencia de las lactonas sesquiterpénicas, se utilizó el revelador de Óleum (solución de ácido sulfúrico, agua y ácido acético en proporción 1:4:20) y posterior calentamiento a $150^{\circ} \mathrm{C}$.

\section{RESULTADOS Y DISCUSIÓN}

\section{Obtención de Extractos y fracciones}

Para determinar el sistema de elución utilizado en el fraccionamiento del extracto en AcOEt sobre columna cromatográfica se ensayaron las mezclas de disolventes $n$ hexano-acetona en distintas proporciones (9:1, 8:2, 7:3, 6:4); en estos sistemas de elución no se observó una separación adecuada de los analitos presentes en el extracto mediante placas de TLC en fase normal, por lo que se ensayaron otros sistemas como AcOEt-AcOH-ácido fórmico-agua (100:11:11:26), AcOEt-MeOH- $\mathrm{H}_{2} \mathrm{O}$ (100:13.5:10), AcOEt-BuOH (95:5, 8:2, 7:3), AcOEt-EtOH (95:5) y AcOEt-BuOH-éter (9:1:0.5). Al no obtener buena separación en los sistemas probados, se procedió a utilizar placas cromatográficas en $\mathrm{RP} 18$ (fase reversa) con el sistema isopropanol-agua (9:1, 8:2, $7: 3$ ), en el cual se produjo una mejor separación en la proporción 9:1.

Para el extracto en AcOEt y las fracciones F1M, F1L, F1N y F1R se tomaron los espectros UV entre los 400 y $200 \mathrm{~nm}$ en metanol, obteniendo como resultado una longitud máxima de absorción a $275 \mathrm{~nm}$ presente en el extracto y sus fracciones, lo que evidencia la posible presencia de compuestos de tipo fenólico como son flavononas, isoflavononas, catequinas y antocianinas, cumarinas con núcleo benzo- $\alpha$-pirona, benzoquinonas y naftoquinonas (Satyajit \& Lutfun, 2012). Este tipo de compuestos pueden encontrarse en su forma glicosilada, lo que les permite mayor solubilidad en disolventes polares y medianamente polares (Harborne, 1973; Yoshida et al., 2010). Los anteriores resultados coinciden con reportes sobre de la presencia de alto porcentaje de compuestos fenólicos en plantas de la familia Melastomataceae (Yoshida et al., 2005; Yoshida et al., 1999). Los resultados de las pruebas de caracterización se presentan en la tabla 1 , donde se evidencia la presencia de taninos de tipo condensado.

Los resultados de las pruebas cualitativas anteriormente mostrados, demuestran la presencia de taninos (principalmente del tipo condensado) y lactonas sesquiterpénicas en las fracciones $\mathrm{F} 1 \mathrm{~L}, \mathrm{~F} 1 \mathrm{M}, \mathrm{F} 1 \mathrm{~N}$ y el extracto en AcOEt, razón por la cual se realizaron pruebas sobre placa cromatográfica para confirmar la presencia de este tipo de compuestos, utilizando como sistema de elución BAW (5:4:1). Como se puede observar en la tabla 2, la fracción F1M presenta un mayor contenido de taninos condensados, ya que se produjo una coloración más intensa, en comparación con las fracciones que dieron positivo para taninos y lactonas sesquiterpénicas.

Algunas especies de la familia melastomataceae han sido estudiadas desde el punto de vista fitoquímico obteniéndose principalmente compuestos del tipo polifenólico, los que en su mayoría corresponden a taninos hidrolizables monoméricos y oligoméricos (Ocampo Serna \& Isaza Martínez, 2015). Hasta el momento no se ha encontrado algún reporte en el que se indique sobre la presencia de taninos en la especie Miconia caudata; por su parte, este sería el primer reporte en el que se determine la presencia de taninos tanto hidrolizables como condensados en esta planta.

Tabla 1. Pruebas químicas cualitativas para la identificación de metabolitos presentes en extracto y en fracciones (Waksmundzka-Hajnos et al., 2008).

\begin{tabular}{ccccccc}
\hline Eruebas & Extracto & F1L & F1M & F1N & F1O & F1
\end{tabular}

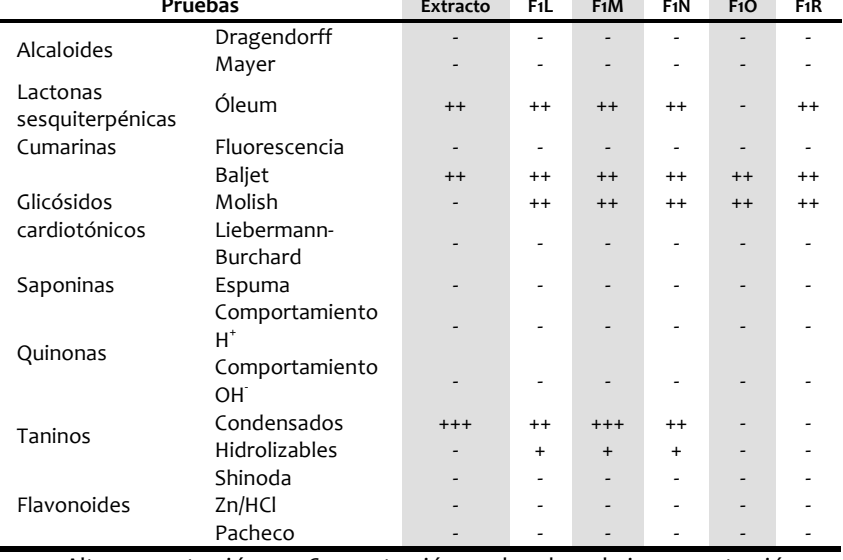

+++: Alta concentración; ++: Concentración moderada; +: baja concentración; -: prueba negativa

Tabla 2. Pruebas realizadas en placa cromatográfica para confirmar presencia de taninos condensados e hidrolizables (Waksmundzka-Hajnos et al., 2008).

\begin{tabular}{cccccc}
\hline & Pruebas & Extracto & F1L & F1M & F1N \\
\hline Vapores de $\mathrm{NH}_{3}$ & Taninos hidrolizables & + & + & + & - \\
Vainillina/ $\mathrm{HCl}$ & Taninos condensados & + & + & + & - \\
$\mathrm{NO}_{2} 10 \%$ & Taninos hidrolizables & + & + & + & - \\
$\mathrm{KIO}_{3}$ sat. & Taninos condensados & + & + & + & - \\
\hline
\end{tabular}

\section{Evaluación de la actividad alelopática}

En la evaluación de la actividad alelopática en el extracto en AcOEt se evidenció un efecto significativo de inhibición en el hipocótilo de las plántulas de L. sativa (fig. 1) con porcentajes de inhibición mayores del 50\%, sugiriendo que este extracto puede actuar como un fuerte inhibidor del crecimiento de las raíces de plantas invasoras. Para el epicótilo se presentó una inhibición moderada (fig. 2), la cual fue disminuyendo con el paso del tiempo, lo que podría expresarse por una disminución en la capacidad de penetración del extracto. 


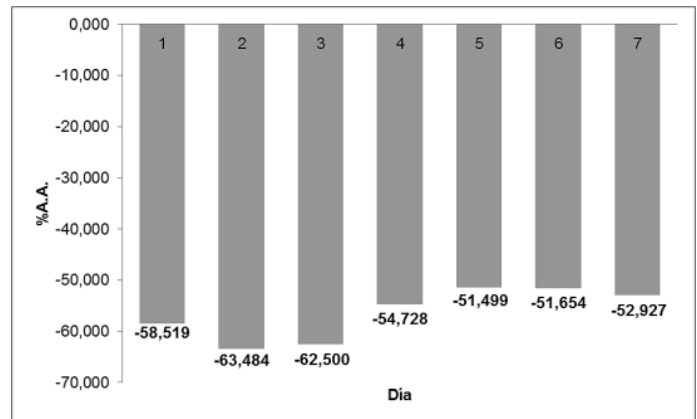

Figura 1. Medida de crecimiento del extracto en AcOEt sobre hipocótilo de las plántulas de L. sativa.

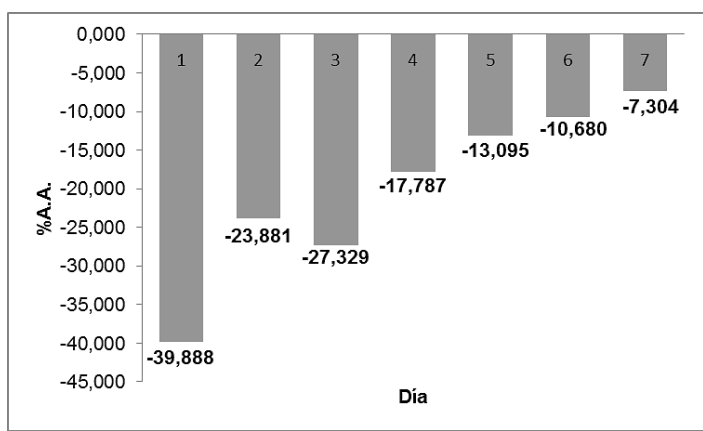

Figura 2. Medida de crecimiento del extracto en AcOEt sobre epicótilo de las plántulas de L. sativa.

Las fracciones F1L y F1N presentaron un efecto inhibidor del crecimiento sobre las plántulas de L. sativa, lo cual podría atribuirse a la presencia de taninos y lactonas sesquiterpénicas como componentes activos mayoritarios. La fracción F1M presentó un comportamiento de estimulación del crecimiento tanto en el epicótilo como en hipocótilo (fig. 3 y 4), estos resultados pueden deberse a la alta concentración de taninos condensados o a la presencia de otros núcleos fitoquímicos. Este efecto es comparable a la acción de las hormonas de crecimiento presentes en las plantas (Macías et al., 2004), por lo que se podría utilizarse como un agente promotor de crecimiento en los cultivos.

Lo anterior es consistente con estudios previos, donde se ha encontrado que algunos polifenoles inhiben la descarboxilación de la hormona de crecimiento IAA (ácido indol acético), por lo que se produce estimulación de crecimiento de las plántulas (Li et al., 2010). Además estudios realizados por el grupo Polifenoles sobre algunas plantas de la familia melastomataceae sugieren que el extracto en isopropanol-agua (65:35) de Henriettella trachyphylla tiene un efecto promotor de crecimiento sobre el epicótilo en plántulas de L. sativa (Isaza-Martínez et al., 2005).

Se seleccionó la fracción F1R para ser separada debido a que presentó el mejor comportamiento alelopático de inhibición con las plántulas de L. sativa, comparando los resultados con las otras fracciones evaluadas. De este fraccionamiento, se obtuvieron 6 subfracciones como son F1R1 (5.10 mg), F1R2 (8.80 mg), F1R3 (1.70 mg), F1R4 (1.80 mg), F1R5 (21.50 mg) y F1R6 (24.00 mg). A cada fracción se le realizó la prueba de Óleum para identificar presencia de lactonas sesquiterpénicas utilizando como sistema de elución $n$-hexano-acetona (7:3). Todas las fracciones dieron prueba positiva para la presencia de lactonas sesquiterpénicas excepto para la fracción F1R1.

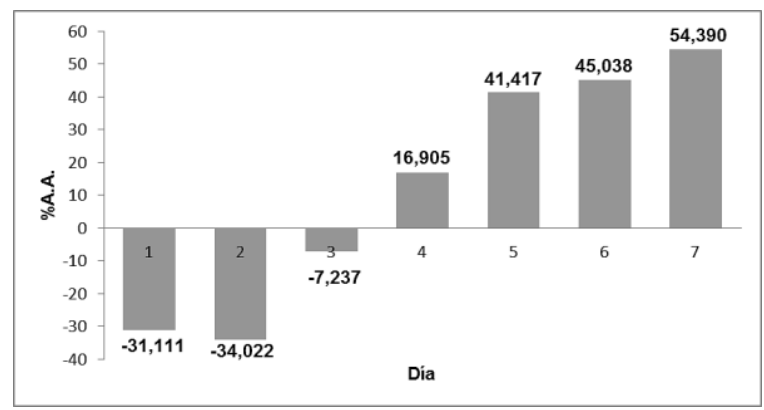

Figura 3. Efecto de la fracción F1M en el crecimiento de hipocótilo.

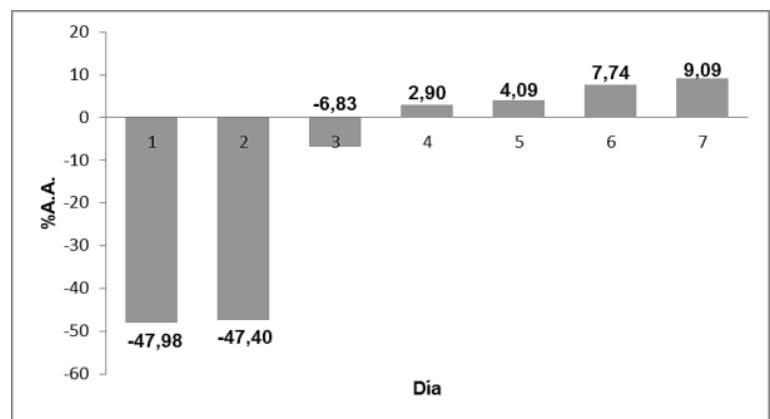

Figura 4. Efecto de la fracción F1M en el crecimiento de epicótilo.

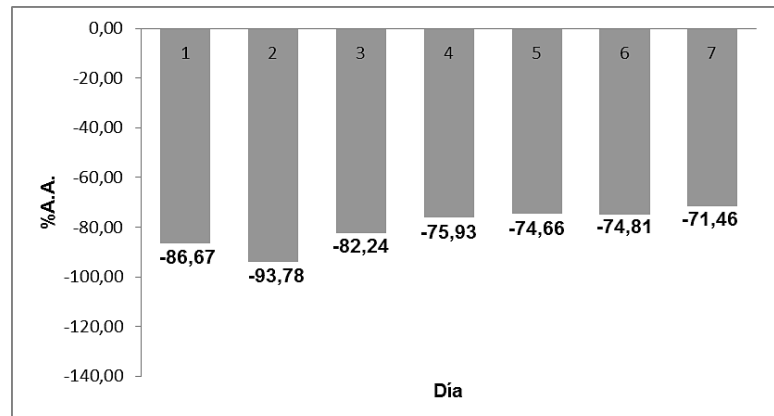

Figura 5. Efecto de la fracción F1R en el crecimiento de hipocótilo.

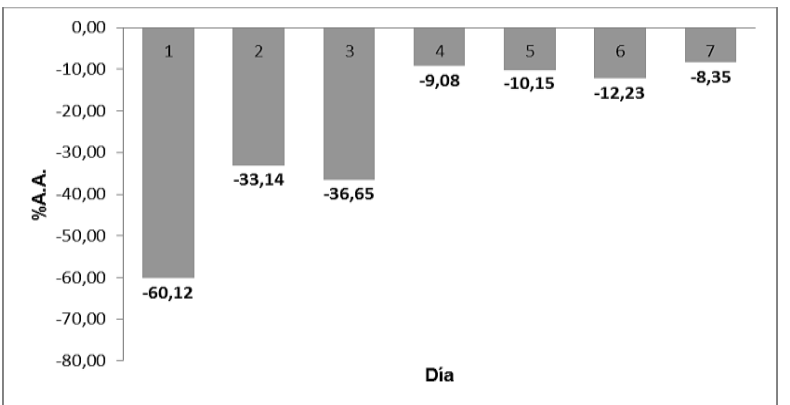

Figura 6. Efecto de la fracción F1R en el crecimiento de epicótilo. 
En el epicótilo después de 24 horas del ensayo se manifiesta un porcentaje de actividad alelopático de inhibición del $-60,12 \%$ y a partir del cuarto día desciende el efecto inhibitorio sobre las plántulas de L. sativa. En la fracción F1R se observó una potente actividad alelopática con efecto inhibidor del crecimiento, principalmente sobre hipocótilo. Según el perfil cromatográfico y análisis fitoquímico solo se identificó la presencia de lactonas sesquiterpénicas. Por tanto, es probable que estas sean las responsables de tal efecto sobre la plántula $L$. sativa. Se ha encontrado en otros estudios, que las lactonas sesquiterpénicas pueden tener actividad regulatoria sobre la germinación y crecimiento de plantas (Mukevji, 2006; Macías et al., 2004; Cutler \& Cutler, 1999). Parece ser que la disponibilidad de grupos que pueden ser alquilados juega un papel importante en la actividad (Rizvi \& Rizvi, 1992).

Otros estudios sobre algunas especies del género Miconia reportan actividad alelopática frente a semillas de tomate Río Grande (Galván et al., 2007). Tal es el caso del extracto en acetona-agua al $70 \%$ de Miconia prasina, que presentó efecto inhibitorio sobre el hipocótilo $(-77,47 \%)$ y el epicótilo $(-62,50 \%)$. Otra especie en la que se encontró efecto inhibitorio fue Miconia aeruginosa en su extracto butanólico, actuando sólo sobre el hipocótilo $(-24,92 \%)$. También se ha reportado que el extracto en AcOEt de Miconia coronata presentó inhibición en el hipocótilo (-91.16\%) y epicótico ($68,20 \%$ ) obtenido a partir del fraccionamiento de la fase acuosa y butanólica (Lopera et al, 2009).

La identificación de los compuestos responsables de la inhibición en el crecimiento de semillas de L. sativa, puede ser aprovechada para generar herbicidas alternativos que por su naturaleza tienen una menor toxicidad que los herbicidas sintéticos (Sampietro et al., 2009). Por tal motivo, se requiere profundizar en el estudio de los constituyentes mayoritarios presentes en el extracto en AcOEt y sus fracciones, para así establecer una relación entre los compuestos presentes en las muestras evaluadas y la actividad presentada.

\section{CONCLUSIONES |}

En este trabajo se determinó que el extracto en AcOEt y sus fracciones poseen una actividad alelopática sobre el hipocótilo y epicótilo de la plántula de L. sativa. Así mismo se determinó que la fracción F1M mostró un efecto estimulante del crecimiento, lo cual puede estar asociado con la alta concentración de taninos condensados.

\section{REFERENCIAS |}

- Cutler, H. G., Cutler, S. J. (1999). Biologically active natural products: agrochemical. Boca Raton: CRC Press.

- Escobar D., L. M., Agudelo R., C., Jiménez-González, F. J., Isaza M., J. H. (2008). Actividad alelopática en maceta del extracto isopropanol-agua (65:35) de Miconia caudata (melastomataceae). Trabajo de grado, programa de Química Industrial, Escuela de Tecnología Química, Facultad de Tecnología, Universidad Tecnológica de Pereira, Pereira, Risaralda. 133 p.

- Galván, J. L., Restrepo, J. C., Jiménez-González, F. J., Isaza M., J. H. (2007). Actividad alelopática de algunas especies de los géneros Miconia, Tibouchina, Henriettella, Tococa, Aciotis y Bellucia (melastomataceae). Scientia et Technica, 33,409-413.

- Harborne, J. H. (1973). Phytochemical methods. Chapman and Hall. 278 p.

- Isaza, J. H., Ito, H., Yoshida, T. (2004). Oligomeric hydrolyzable tannins from Monochaetum multiflorum. Phytochemystry, 65,359-367.

- Isaza-Martínez, J. H. Jiménez-González, F. J., Veloza, L. A., Ramírez, L. S. (2005). Actividad alelopática de Henriettella trachyphylla (melastomatácea). Actualidades Biológicas, supl. 1,17-20.

- Li, H. Z., Wang, Q., Ruan, Z., Pan, C. D., Jiang, D. A. (2010). Phenolics and plant allelopathy. Molecules, 15,8933-8952.

- Lopera, I. A., Velásquez, C. D., Ramírez A., L. S., Isaza, J. H. (2009). Actividad ictiotóxica de extractos polares y apolares de algunas especies de los géneros Miconia, Clidemia (Melastomataceae) y Palicourea, Hamelia (Rubiaceae). Trabajo de grado, programa de Química Industrial, Escuela de Tecnología Química, Facultad de Tecnología, Universidad Tecnológica de Pereira, Pereira, Risaralda. $149 \mathrm{p}$.

- Macías, F. A., Galindo, J. C. G., Molinillo, J. M. G., Cutler, H. G. (2004) Allelopathy chemistry and mode of actions of allelochemicals. CRC Press, $372 \mathrm{p}$.

- Macías, F. A., G. Molinillo, J. M., Varela, R. M., G. Galindo, J. C. (2007) Allelopathy - a natural alternative for weed control. Pest Management Science, 63,327-348.

- Mukevji, K. G. (2006). Allelochemicals: biological control of plant pathogens and diseases. Netherland: Springer.

- Ocampo Serna, D. M., Isaza Martínez, J. H. (2015). Phenolics and polyphenolics from Melastomataceuos Species. Molecules, 20(10),1781817847.

- Rice, E. L. (1984). Allelopathy. New York: Academic Press.

- Rizvi, S. J., Rizvi, V. (1992). Allelopathy: basic and applied aspects. Springer, $480 \mathrm{p}$.

- Sampietro, D. A., Catalán, A. N., Vattuone, M. A. (2009). Isolation, identification and characterization of allelochemicals-Natural products. USA: Science Publishers.

- Satyajit, D. S., Lutfun, N. (2012). Natural products isolation: methods and protocols. New York: Humana Press.

- Schofield, P., Mbugua, D. M., Pell, A. N. (2001). Analysis of condensed tannins: a review. Animal Feed Science and Technology, 91,21-40.

- Waksmundzka-Hajnus, M., Sherman, J., Kowalska, T. (2008). Thin layer chromatography in phytochemistry. Boca Raton: CRC Press.

- Yoshida, T., Amakura, Y., Yokura, N. Ito, H., Isaza, J. H., Ramírez, S., Pelaez, D., Renner, S. S. (1999). Oligomeric hydrolysable tannins from Tibouchina multiflora. Phytochemistry, 52,1661-1666.

- Yoshida, T., Amakura, Y., Yoshimura, M. (2010). Structural features and biological properties of ellagitannins in some plant families of the Order Myrtales. International Journal of Molecular Sciences, 11,79-106.

- Yoshida, T., Ito, H., Isaza, J. H. (2005). Pentameric ellagitannins in melastomataceous plants - chemotaxonomic significance. Phytochemistry, 66(17),1972-1983. 